\title{
Prognostic significance of regulatory $T$ lymphocytes in patients with hepatocellular carcinoma
}

\author{
Ai-bin ZHANG ${ }^{1,2,3,4}$, Yi-gang QIAN ${ }^{1,2,3,4}$, Shu-sen $Z_{H E N G}{ }^{\dagger 1,2,3,4}$ \\ ( ${ }^{1}$ Division of Hepatobiliary and Pancreatic Surgery, Department of Surgery, the First Affiliated Hospital, \\ School of Medicine, Zhejiang University, Hangzhou 310003, China) \\ ( ${ }^{2}$ Key Laboratory of Combined Multi-organ Transplantation, Ministry of Public Health, Hangzhou 310003, China) \\ ( ${ }^{3}$ Key Laboratory of Organ Transplantation, Zhejiang Province, Hangzhou 310003, China) \\ $\left({ }^{4}\right.$ Collaborative Innovation Center for Diagnosis Treatment of Infectious Diseases, Hangzhou 310003, China) \\ †E-mail: shusenzheng@zju.edu.cn \\ Received June 25, 2016; Revision accepted Aug. 26, 2016; Crosschecked Nov. 10, 2016
}

\begin{abstract}
We investigated the prognostic role of regulatory $T$ cells (Tregs) in patients with hepatocellular carcinoma (HCC). Relevant evidence regarding prognostic significance of Tregs was systematically searched in MEDLINE and Embase databases. A meta-analysis was performed to compare survival in patients with high or low Tregs level (either in peripheral blood or tumor). Eighteen studies were identified that fulfilled for the eligibility criteria and were included for data synthesis. Our pooled hazard ratios (HRs) demonstrated that increased Tregs intratumoral accumulation was significantly associated with worse overall survival $(\mathrm{HR}=2.04,95 \%$ confidence interval $(\mathrm{Cl}): 1.72-2.42)$ and diseasefree survival ( $\mathrm{HR}=1.82,95 \% \mathrm{Cl}: 1.58-2.09)$. Three studies evaluated the role of Tregs in peripheral blood, and all of them showed that increased peripheral Tregs correlated with shortened disease-free and overall survival. Collectively, our results showed that the increased Tregs count is tightly associated with the shortened survivals. Its measurement in either primary tumor or even circulation might be a candidate marker of prognostic significance in HCC patients.
\end{abstract}

Key words: Hepatocellular carcinoma, Regulatory T cells, Prognosis http://dx.doi.org/10.1631/jzus.B1600264

CLC number: R735

\section{Introduction}

Hepatocellular carcinoma (HCC) is the most common primary malignancy of the liver, representing the third leading cause of cancer-related death worldwide (Jemal et al., 2011). Its overall dismal prognosis is a result of high incidence of metastasis and postoperative recurrence, in particular the intrahepatic spread (Poon et al., 2000). Both aberrant transformation of tumor cells themselves and evolution of surrounding microenvironment are believed to

\footnotetext{
${ }^{\ddagger}$ Corresponding author

* Project supported by the National Natural Science Foundation of China (No. 81370644) and the Zhejiang Provincial Natural Science Foundation of China (No. LY17H60026)

(b) ORCID: Ai-bin ZHANG, http://orcid.org/0000-0002-1149-4347

(C) Zhejiang University and Springer-Verlag Berlin Heidelberg 2016
}

contribute to disease progression (Hernandez-Gea et al., 2013).

The immune cells are abundant in HCC stroma. Among the stromal cells, the population of immunosuppressive cells like tumor-associated macrophages, myeloid-derived suppressive cells, and regulatory $\mathrm{T}$ cells (Tregs) facilitates the evasion of tumor cell clearance by $\mathrm{CD}^{+}$cytotoxic lymphocytes (CTLs). In particular, Tregs, a subgroup of $\mathrm{CD}^{+}$ T-helper cells, constitute a critical component in modulating local immune microenvironment (Sakaguchi, 2000; Shevach, 2002). It has been shown that the number of Fox $3^{+}$Tregs markedly increased in both peripheral blood and tumor of HCC patients, which is linked to uncontrolled tumor growth and progression (Ormandy et al., 2005; Unitt et al., 2005). On the other hand, a few studies have demonstrated 
that Tregs play a rather minor role in HCC compared to other immune cells.

Thus, the present study sought to explore the prognostic significance of Tregs on prognosis among HCC patients by systematic review and meta-analysis.

\section{Materials and methods}

\subsection{Search strategy and study selection}

The Embase and MEDLINE databases were electronically searched for the identification of pertinent studies till the end of February 2016. For MEDLINE search, the mesh terms "T-Lymphocytes", "Regulatory", and "Carcinoma, Hepatocellular" were used, while "Regulatory T cells", "Tregs", and "Hepatocellular carcinoma" were used for the Embase database. The language was restricted to English Reference lists of identified primary articles were further screened to find missed studies during the electronic search. All candidate articles were initially screened and cross-checked by two independent reviewers for inclusion of eligible studies. Full-text review was performed when decision could not be reached based on titles and abstracts, and the discrepancies were resolved by discussion with a third investigator.

\subsection{Inclusion criteria}

The inclusion criteria for eligible studies consisted of the following: (1) patients with proven diagnosis of HCC; (2) measurement of Tregs either in circulation, peritumoral, or intratumoral area; and (3) investigation of effect of Tregs on overall survival (OS) or disease-free survival (DFS). Studies not directly reporting hazard ratios (HRs) were allowed only if the required data were available for statistical estimation as described below. When articles came from the same research group, duplicate patient populations were carefully evaluated mainly through study period, hospital, and treatment information. If identical patient populations were studied, only the one containing the most complete information was taken into account.

\subsection{Data extraction}

Data were extracted independently by two individuals. The required data were predetermined and were as follows: general study information (authors, year of publication, and type of study design); patient clinical data (number of patients studied, gender, tumor stage, and treatment modality); estimation of Tregs count (location, methods of measurement, and cutoff levels determining "high" or "low" Tregs count); data regarding OS and DFS (HR or relevant data can be used to calculate HR); information concerning quality assessment (patient selection, study comparability, outcome of interest, and follow-up).

\subsection{Quality assessment}

Quality of the methodology for each of the enrolled articles was rated using the Newcastle-Ottawa Quality Assessment Scale for cohort studies, which is based on three aspects of study design including selection, comparability, and outcome (Will and Steidl, 2014). One star was awarded if certain criterion was met, and the possible total star points ranged from 0 to 9. We adopted similar predefined principals as previous review during quality evaluation (Schoenleber et al., 2009).

\subsection{Statistical analysis}

For data synthesis, the primary outcome was OS or DFS in patients with high Tregs count (either in circulatory system or intratumoral area) as compared with those having a low number. Similarly, the survival comparison was also carried out for balance between Tregs and $\mathrm{CD} 8^{+}$lymphocytes, commonly shown as the Tregs $/ \mathrm{CD}^{+}$ratio. All survival data were expressed as HR with $95 \%$ confidence interval (CI). When HR was not reported directly in articles, an estimate was made on the basis of established methods including extracting data from reported survival curves (Tierney et al., 2007).

The heterogeneity of primary result was appraised initially with the chi-square test and then quantified using $I^{2}$ statistics. If heterogeneity was present among combined studies, random effect model was adopted, and if not, data were pooled with fixed effect model. A $P$-value below 0.05 was believed to be significant. As for evaluation of publication bias, funnel plot analysis was performed and Egger's test was not applied, given the small number of primary studies. STATA 10 (STATA Corp., LP, USA) was used to generate forest plots of combined HRs with 95\% CIs. 


\section{Results}

\subsection{Study characteristics}

Our search strategy yielded 573 primary articles, of which 16 were eventually identified fulfilling the eligibility criteria (Fu et al., 2007; Gao et al., 2007; 2009; Kobayashi et al., 2007; Sasaki et al., 2008; Cai et al., 2009; Ju et al., 2009a; 2009b; Zhou et al., 2009; 2010; Chen et al., 2011; Shen et al., 2011; Huang et al., 2012; Mathai et al., 2012; Wang et al., 2012; Lin et al., 2013) (Fig. 1). Several of the studies included were conducted by the same research groups, but the patient cohorts were found to be not duplicated through careful examinations. All included studies are cohortbased design, and the patients' characteristics are shown in Table 1. Among them, 14 analyses had data regarding Tregs in tumor, while 3 cohort studies investigated those in peripheral blood. Of note, the patient population in most of included studies underwent liver resection as first treatment strategy.

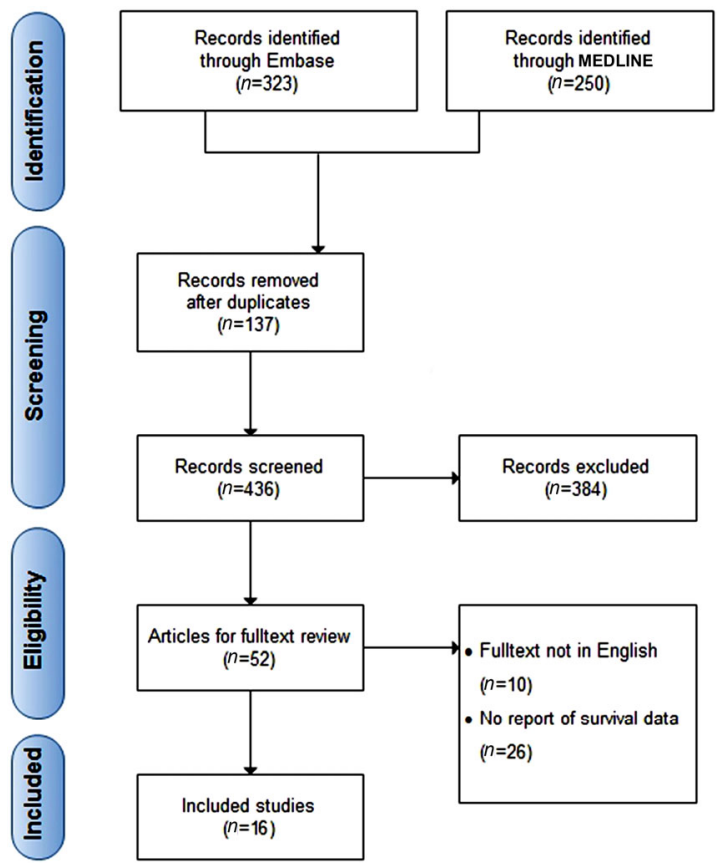

Fig. 1 Flowchart for selection of studies

Table 1 Patients' characteristics of included studies

\begin{tabular}{|c|c|c|c|c|c|c|}
\hline Study & Study design & $\begin{array}{c}\text { Number of } \\
\text { patients }(\mathrm{M} / \mathrm{F})\end{array}$ & $\begin{array}{c}\text { Aetiology } \\
\text { (HBV/HCV/ } \\
\text { both/none) }\end{array}$ & $\begin{array}{c}\text { TNM I/II } \\
(\mathrm{III} / \mathrm{IV})\end{array}$ & Treatment & $\begin{array}{c}\text { Follow-up } \\
\text { duration }^{*} \text { (month) }\end{array}$ \\
\hline \multicolumn{7}{|l|}{ Liver-resident Tregs } \\
\hline Gao et al., 2007 & Retrospective, cohort & $302(260 / 42)$ & $241 / 1 / 14 / 46$ & $237(65)$ & Resection & 58 (median) \\
\hline Kobayashi et al., 2007 & Retrospective, cohort & $147(113 / 34)$ & $47 / 79 / 9 / 12$ & $110(37)$ & Resection & 52.8 \\
\hline Sasaki et al., 2008 & Retrospective, cohort & $164(126 / 38)$ & $37 / 85 / \mathrm{ns} / \mathrm{ns}$ & ns & Resection & 63.5 \\
\hline Shen et al., 2011 & Prospective, cohort & $76(70 / 6)$ & $70 / \mathrm{ns} / \mathrm{ns} / \mathrm{ns}$ & $51(25)$ & Resection & ns \\
\hline Chen et al., 2011 & Retrospective, cohort & $143(125 / 18)$ & $120 / \mathrm{ns} / \mathrm{ns} / \mathrm{ns}$ & $95(48)$ & Resection & ns \\
\hline Huang et al., 2012 & Prospective, cohort & $54(45 / 9)$ & $43 / \mathrm{ns} / \mathrm{ns} / \mathrm{ns}$ & $23(31)$ & Resection & 44 \\
\hline Mathai et al., 2012 & Retrospective, cohort & $91(72 / 19)$ & $63 / 18 / 23 / \mathrm{ns}$ & ns & $\begin{array}{c}\text { OLT/resection } \\
(74 / 17)\end{array}$ & ns \\
\hline Wang et al., 2012 & Prospective, cohort & $137(112 / 25)$ & $137 / \mathrm{ns} / \mathrm{ns} / \mathrm{ns}$ & $88(49)^{\S}$ & $\begin{array}{l}\text { Resection/ } \\
\text { AT/TACE } \\
(34 / 55 / 48)\end{array}$ & 27.5 (median) \\
\hline Ju et al., 2009a & Prospective, cohort & $130(112 / 18)$ & $111 / \mathrm{ns} / \mathrm{ns} / \mathrm{ns}$ & $74(56)^{\#}$ & Resection & 31.8 \\
\hline Cai et al., 2009 & Retrospective, cohort & $173(147 / 26)$ & $157 / 1 / 3 / 12$ & $76(97)^{\S}$ & Resection & 37.2 \\
\hline Ju et al., 2009b & Retrospective, cohort & $207(173 / 34)$ & $192 / 2 / 3 / 10$ & 98 (109) & Resection & 27.9 \\
\hline Zhou et al., 2009 & Retrospective, cohort & $131(113 / 18)$ & $118 / \mathrm{ns} / \mathrm{ns}$ & $94(37)$ & Resection & ns \\
\hline Gao et al., 2009 & Retrospective, cohort & $240(204 / 36)$ & $219 / 1 / 4 / 16$ & $182 / 58$ & Resection & ns \\
\hline Lin et al., 2013 & ns & $245(\mathrm{~ns})$ & ns & ns & Resection & ns \\
\hline \multicolumn{7}{|l|}{ Circulating Tregs } \\
\hline Wang et al., 2012 & Prospective, cohort & $101(83 / 18)$ & $101 / \mathrm{ns} / \mathrm{ns}$ & $67(34)^{\S}$ & $\begin{array}{l}\text { Resection/ } \\
\text { AT/TACE } \\
(21 / 44 / 36)\end{array}$ & ns \\
\hline Zhou et al., 2010 & Prospective, cohort & $49(41 / 8)$ & $38 / \mathrm{ns} / \mathrm{ns}$ & $49(0)^{\S}$ & $\mathrm{AT}$ & ns \\
\hline Fu et al., 2007 & ns & $75(\mathrm{~ns})$ & $75 / \mathrm{ns} / \mathrm{ns}$ & ns & $\begin{array}{l}\text { Without } \\
\text { treatment }\end{array}$ & ns \\
\hline
\end{tabular}

${ }^{*}$ Data are expressed as mean unless specified; ${ }^{\S} \mathrm{BCLC}$ staging $0 / \mathrm{A}(\mathrm{B} / \mathrm{C}) ;{ }^{\#} \mathrm{TNM}$ stage I (II/III). OLT: orthotopic liver transplantation; AT: ablation therapy; TACE: transcatheter arterial chemoembolization; ns: not stated 
Besides, one investigation mainly focused on those receiving liver transplantation, while there were two studies that enrolled patients undergoing transcatheter arterial chemoembolization or ablation therapy. In addition, Fu et al. (2007) studied patients who did not receive any antitumor therapy.

\subsection{Quality evaluation of included studies}

The points acquired by each of enrolled studies are shown in Table 2. In detail, there were no analyses intended to control for potential confounding factors. Except for the category of comparability, most studies lack points for evaluation of outcomes including non-blinded outcome assessment and follow-up inadequacy. In particular, a fraction of cohort studies that were retrospective in design only analyzed patients with sufficient survival record, which was believed to be unqualified obtaining point for the item of adequacy of follow-up.

\subsection{Summary estimates of primary studies}

There were 10 studies evaluating the prognostic role of intratumoral Tregs level in HCC. There was no remarkable heterogeneity between pooled HRs for either OS $\left(I^{2}=31.6 \%, P=0.147\right)$ or DFS $\left(I^{2}=12.4 \%\right.$,
$P=0.323$ ), and thus fixed-effect model was used in both analyses. Our pooled HRs showed that increased Tregs intratumoral accumulation was significantly associated with worse OS (HR $=2.04,95 \%$ CI: 1.72-2.42) and DFS (HR=1.82, 95\% CI: $1.58-2.09)$ (Fig. 2). Among these 10 studies, 8 used absolute FoxP3 $^{+}$cell count to define the Tregs level, while other 2 used percentage of $\mathrm{FoxP}_{3}{ }^{+}$cell among $\mathrm{CD} 4{ }^{+}$ cells as a way of measurement (Table 3). Therefore, subgroup analysis was performed for studies using different ways of measurement for Tregs level. For those using absolute Tregs cell count, a similarly negative effect on prognosis was noted for high Tregs level (OS: $\mathrm{HR}=1.99,95 \%$ CI 1.76-2.47; DFS: $\mathrm{HR}=1.91,95 \%$ CI 1.67-2.16). Quantitative analysis was not done for studies using proportion of $\mathrm{FoxP}^{+} / \mathrm{CD}^{+}$because only two studies were available. However, both of them showed that a high percentage of Tregs correlated with shortened survival in their own analysis.

In addition, we investigated the prognostic significance of balance between tumor-infiltrating Tregs and $\mathrm{CD} 8^{+}$cells. Because of the small number of recruited primary studies, combined quantitative analysis was not performed. In total, three studies determined

Table 2 Quality evaluation for included studies

\begin{tabular}{|c|c|c|c|c|}
\hline & Selection & Comparability & Outcome & \\
\hline Study & $\begin{array}{l}\text { (1) Representativeness of exposed cohort } \\
\text { (2) Selection of non-exposed cohort } \\
\text { (3) Ascertainment of exposure } \\
\text { (4) Demonstration that the outcome of } \\
\text { interest was not present at the start } \\
\text { of the study }\end{array}$ & $\begin{array}{l}\text { (1) Comparability } \\
\text { of cohorts on } \\
\text { the basis of the } \\
\text { design or } \\
\text { analysis }\end{array}$ & $\begin{array}{l}\text { (1) Assessment of outcome } \\
\text { (2) Was follow-up long enough } \\
\text { for outcomes to occur? } \\
\text { (3) Adequacy of follow-up of } \\
\text { cohorts }\end{array}$ & $\begin{array}{c}\text { Total } \\
\text { points }\end{array}$ \\
\hline Gao et al., 2007 & $* 1), * 2), * 3), * 4)$ & none & *2) & 5 \\
\hline $\begin{array}{l}\text { Kobayashi et al., } \\
2007\end{array}$ & $* 1), * 2), * 3), * 4)$ & none & $* 2), * 3)$ & 6 \\
\hline Sasaki et al., 2008 & $* 1), * 2), * 3), * 4)$ & none & $* 2), * 3)$ & 6 \\
\hline Shen et al., 2011 & $* 1), * 2), * 3), * 4)$ & none & *3) & 5 \\
\hline Chen et al., 2011 & $* 3), * 4)$ & none & *2) & 3 \\
\hline Huang et al., 2012 & $* 1), * 2), * 3), * 4)$ & none & $* 1), * 2), * 3)$ & 7 \\
\hline Mathai et al., 2012 & $* 1), * 2), * 3), * 4)$ & none & $* 2), * 3)$ & 6 \\
\hline Wang et al., 2012 & $* 1), * 2), * 3), * 4)$ & none & $* 2), * 3)$ & 6 \\
\hline Zhou et al., 2010 & $* 2), * 3), * 4)$ & none & *3) & 4 \\
\hline Fu et al., 2007 & $* 1), * 2), * 3), * 4)$ & none & none & 4 \\
\hline Ju et al., 2009a & $* 1), * 2), * 3), * 4)$ & none & $* 2)$ & 5 \\
\hline Cai et al., 2009 & $* 1), * 2), * 3), * 4)$ & none & $* 2), * 3)$ & 6 \\
\hline Ju et al., 2009b & $* 3), * 4)$ & none & $* 2)$ & 3 \\
\hline Zhou et al., 2006 & $* 1), * 2), * 3), * 4)$ & none & none & 4 \\
\hline Gao et al., 2009 & $* 1), * 2), * 3), * 4)$ & none & none & 4 \\
\hline Lin et al., 2013 & $* 1), * 2), * 3), * 4)$ & none & none & 4 \\
\hline
\end{tabular}


(a)

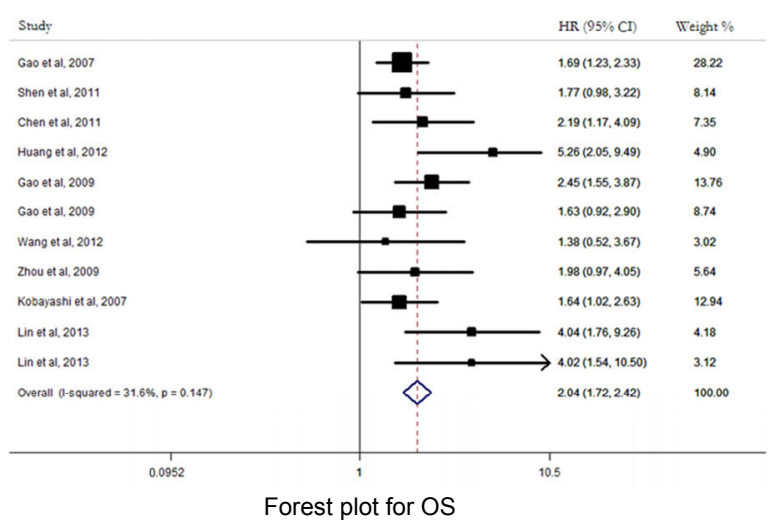

(b)

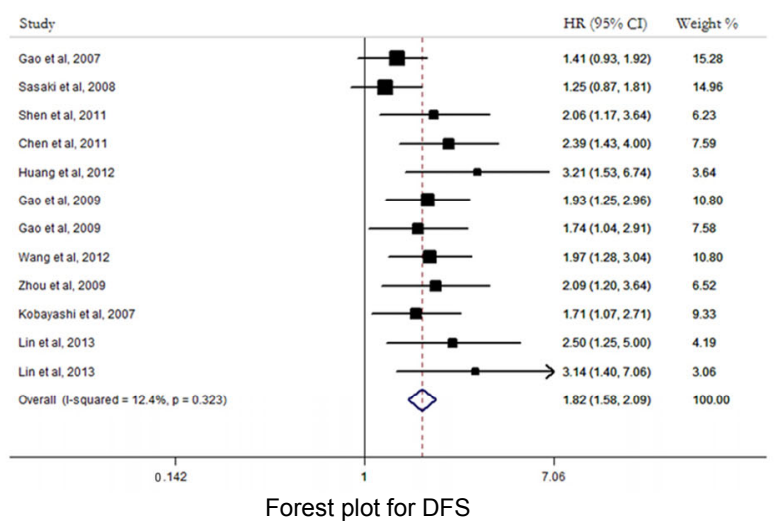

Fig. 2 Forest plot suggesting that intratumoral Tregs count was associated with OS (a) and DFS (b) of HCC patients

Table 3 Extracted information for each included study

\begin{tabular}{llclc}
\hline \multicolumn{1}{c}{ Study } & Identification method & High/low Tregs patients & HR for DFS & HR for OS \\
\hline Intratumoral Tregs & & & & \\
Gao et al., 2007 & FoxP3 $^{+}$count & $147 / 155$ & Reported in text & Reported in text \\
Kobayashi et al., 2007 & FoxP3 $^{+} / \mathrm{CD}^{+}$proportion & $73 / 74$ & Reported in text & Reported in text \\
Sasaki et al., 2008 & FoxP3 $^{+}$count & $84 / 80$ & Survival curves & $\mathrm{ns}$ \\
Shen et al., 2011 & FoxP3 $^{+}$count & $35 / 41$ & Survival curves & Survival curves \\
Chen et al., 2011 & FoxP3 $^{+}$count & $57 / 86$ & Reported in text & Reported in text \\
Huang et al., 2012 & FoxP3 $^{+}$count & $27 / 27$ & Reported in text & Reported in text \\
Huang et al., 2012 & FoxP3 $^{+} / \mathrm{CD}^{+}$ratio & $\mathrm{ns}$ & Reported in text & Reported in text \\
Mathai et al., 2012 & FoxP3 $^{+} / \mathrm{CD}^{+}$ratio & $\mathrm{ns}$ & Reported in text & Reported in text \\
Wang et al., 2012 & FoxP3 $^{+}$count & $68 / 69$ & Reported in text & Survival curves \\
Cai et al., 2009 & FoxP3 $^{+} / \mathrm{CD}^{+}$ratio & $\mathrm{ns}$ & Reported in text & Reported in text \\
Zhou et al., 2009 & FoxP3 $^{+}$count & $36 / 49$ & Reported in text & Reported in text \\
Gao et al., 2009 & FoxP3 $^{+}$count & $\mathrm{ns}$ & Reported in text & Reported in text \\
Lin et al., 2013 & FoxP3 $^{+} / \mathrm{CD}^{+}$proportion & $\mathrm{ns}$ & Reported in text & Reported in text \\
Circulating Tregs & & & & \\
Wang et al., 2012 & FoxP3 $^{+} / \mathrm{CD}^{+}$proportion & $50 / 51$ & Survival curves & Reported in text \\
Zhou et al., 2010 & FoxP3 $^{+} / \mathrm{CD}^{+}$proportion & $59 / 52$ & Reported in text & ns \\
Fu et al., 2007 & FoxP3 $^{+} / \mathrm{CD}^{+}$proportion & $31 / 44$ & Survival curves & ns \\
\hline
\end{tabular}

ns: not stated

the role of $\mathrm{FoxP}^{+} / \mathrm{CD}^{+}$ratio and, consistently, a better DFS and OS were observed in groups with a lower ratio. In contrast, another study that divided patients into four groups based on combination of Tregs and $\mathrm{CD} 8^{+}$cell counts found similar survival outcomes among different groups.

There were only three studies examining Tregs in peripheral blood, and the pooled HRs was not calculated because of the small number of studies (Table 3). All analyses showed that increased peripheral Tregs correlated with shortened DFS and OS. In addition, peritumoral Tregs were evaluated in five investigations, but the limited available survival data excluded a quantitative pooled analysis. Among them, only one study reported an increased risk of death or recurrence for patients with high peritumoral Tregs number, whereas other studies did not find any significant correlations.

Of note, the majority of patients included in our analysis had chronic hepatitis B virus (HBV) infection. There were two studies containing a decent number of patients with hepatitis $\mathrm{C}$ virus (HCV), but they did not address the prognostic role of Tregs exclusively in $\mathrm{HCV}$ patients. In fact, both of them found that high Tregs level was significantly associated with decreased survival in their own analyses. By 
excluding these two studies, the subgroup analysis consistently demonstrated an inverse correlation between Tregs level and survival (OS: HR $=2.13,95 \%$ CI 1.81-2.54; DFS: HR=1.98, 95\% CI 1.73-2.21).

\subsection{Publication bias assessment}

Publication bias was examined through visual assessment of funnel plot and the Egger's test. There were no bias for OS and DFS analyses (Fig. 3).

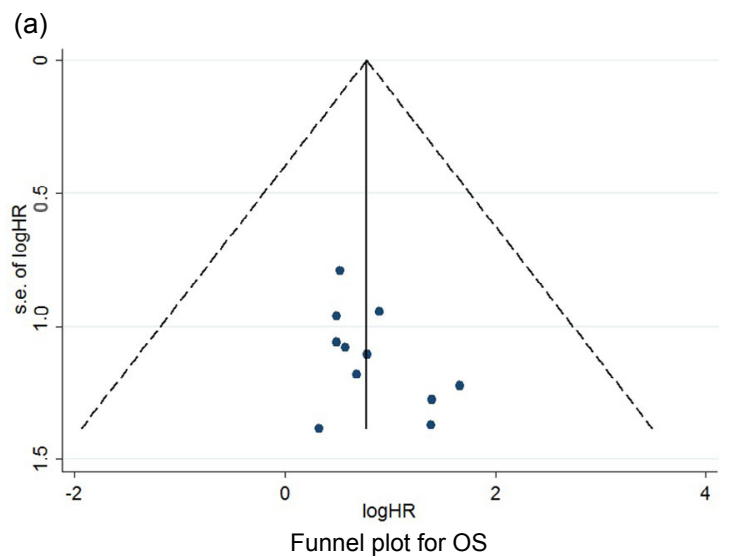

(b)

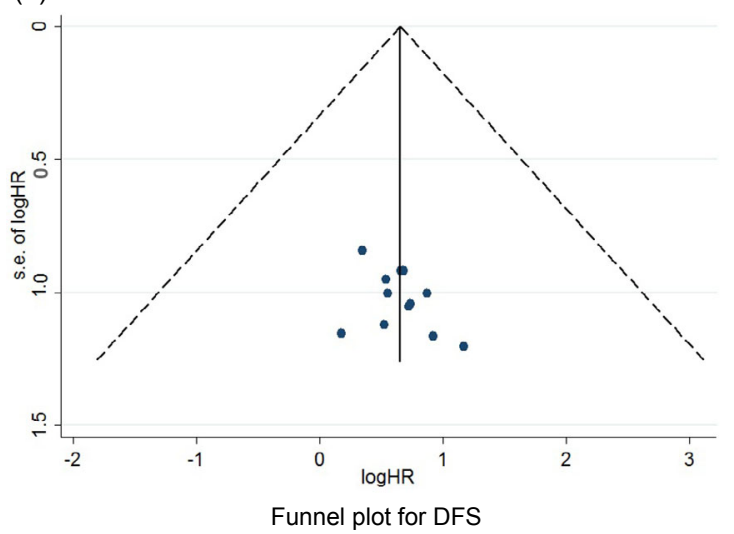

Fig. 3 Evaluation of publication bias through funnel plot for OS (a) and DFS (b)

\section{Discussion}

Currently, there is great interest to investigate the interaction between host immune response and cancer (Lan et al., 2015), with objective of identifying immune markers that could predict clinical outcome and searching for effective immunotherapeutic interventions. The present analysis provides the first systematic review and meta-analysis of studies ex- ploring the prognostic effect of Tregs on patients inflicted by HCC. Summary data showed that high circulating and tumor-infiltrating Tregs levels are associated with decreased OS and DFS.

In this study, we analyzed Tregs in different compartments including peripheral blood and tumor. Intratumoral Tregs represent the forefront interacting with tumor cells and prevent their elimination from antitumor immunity. Compared with its counterparts in peripheral blood, Tregs within tumor bed exhibited more prominent sequestration and superiority in function as well, which are able to impede local tumor outgrowth. On the other hand, Tregs in circulatory system is believed to be an indirect marker of intratumoral accumulation, though their further proliferation within the liver might cause discrepancy in quantity between different locations. Indeed, our pooled data indicate that both of them are significant prognostic factors for predicting long-term survival. Of note, as for evaluation of circulating Tregs, only three studies were eligible for quantitative synthesis and one study with negative result was excluded due to inadequate data. There are several benefits in using peripheral Tregs. First, its examination is done by a simple noninvasive procedure. Second, for patients who do not undergo surgery like resection or transplantation, peripheral Tregs can be measured to assess clinical outcomes. Third, compared with the heterogeneity during interpretation of tissue staining in assessment of tissue Tregs, the lymphocyte count in blood can be accurately and reliably measured.

When evaluating significance of intratumoral Tregs, various indicators could be used. The absolute Tregs count is the most commonly employed method, but different cutoff values defined in each study may cause difficult interpretation. Tregs proportion among $\mathrm{CD}^{+}$lymphocytes is also widely measured, and our two included primary studies adopted this value. Interestingly, Lin et al. (2013) reported that proportion of FoxP $3^{+}$cells but not its absolute number was negatively related to prognosis. When excluding studies using Tregs proportion instead of count, increased Tregs accumulation remained associated with shortened survival. The measurement of the Tregs $/ \mathrm{CD}^{+}$ ratio takes into account the cytotoxic immune response and indicates the balance between these two groups of lymphocytes. Its elevation consistently exhibited a trend for unfavorable prognosis. 
It is noteworthy that most of our included studies were implemented among Asia population, especially China. Therefore, patients were mostly HBV-positive, raising the question concerning validity of results to those HCCs with other underlying etiologies. There are lines of evidence suggesting that HBV infection could modulate host immune reaction through interaction with Tregs, which in turn contribute to viral persistence (Stross et al., 2012). Indeed, it has been demonstrated that Tregs determined HBV patient prognosis through impairing immune response and promoting infection progression (Xu et al., 2006; Peng et al., 2008). More data regarding patients in Western countries are required to further confirm our results.

In the present meta-analysis, we adopted the Newcastle-Ottawa quality scale to assess the primary studies, which is commonly chosen for determining quality of cohort and case-control studies. In this scale, there seems to be no established scoring system to define a study with high or low quality for prognostic analysis of cancer patients. Previous review exploring disease prevalence considered studies with five or more points as high quality, while smaller than four points are believed to be of low quality (Das et al., 2014). In fact, of the 16 included studies, 7 were found to have a score of 5 or more.

In conclusion, currently available evidence supports that increased serum and tissue Tregs counts predict a worse overall and recurrence-free survival. Thus, it is appropriate to use Tregs as a promising prognostic marker for HCC patients.

\section{Compliance with ethics guidelines}

Ai-bin ZHANG, Yi-gang QIAN, and Shu-sen ZHENG declare that they have no conflict of interest.

This article does not contain any studies with human or animal subjects performed by any of the authors.

\section{References}

Cai, M.Y., Xu, Y.F., Qiu, S.J., et al., 2009. Human leukocyte antigen-G protein expression is an unfavorable prognostic predictor of hepatocellular carcinoma following curative resection. Clin. Cancer Res., 15(14):4686-4693. http://dx.doi.org/10.1158/1078-0432.CCR-09-0463

Chen, K.J., Lin, S.Z., Zhou, L., et al., 2011. Selective recruitment of regulatory T cell through CCR6-CCL20 in hepatocellular carcinoma fosters tumor progression and predicts poor prognosis. PLOS ONE, 6(9):e24671. http://dx.doi.org/10.1371/journal.pone.0024671
Das, S.L., Singh, P.P., Phillips, A.R., et al., 2014. Newly diagnosed diabetes mellitus after acute pancreatitis: a systematic review and meta-analysis. Gut, 63(5):818-831. http://dx.doi.org/10.1136/gutjnl-2013-305062

Fu, J., Xu, D., Liu, Z., et al., 2007. Increased regulatory T cells correlate with CD8 T-cell impairment and poor survival in hepatocellular carcinoma patients. Gastroenterology, 132(7):2328-2339.

http://dx.doi.org/10.1053/j.gastro.2007.03.102

Gao, Q., Qiu, S.J., Fan, J., et al., 2007. Intratumoral balance of regulatory and cytotoxic $\mathrm{T}$ cells is associated with prognosis of hepatocellular carcinoma after resection. $J$. Clin. Oncol., 25(18):2586-2593. http://dx.doi.org/10.1200/JCO.2006.09.4565

Gao, Q., Wang, X.Y., Qiu, S.J., et al., 2009. Overexpression of PD-L1 significantly associates with tumor aggressiveness and postoperative recurrence in human hepatocellular carcinoma. Clin. Cancer Res., 15(3):971-979. http://dx.doi.org/10.1158/1078-0432.CCR-08-1608

Hernandez-Gea, V., Toffanin, S., Friedman, S.L., et al., 2013. Role of the microenvironment in the pathogenesis and treatment of hepatocellular carcinoma. Gastroenterology, 144(3):512-527. http://dx.doi.org/10.1053/j.gastro.2013.01.002

Huang, Y., Wang, F.M., Wang, T., et al., 2012. Tumorinfiltrating FoxP3+ Tregs and CD8+ T cells affect the prognosis of hepatocellular carcinoma patients. Digestion, 86(4):329-337.

http://dx.doi.org/10.1159/000342801

Jemal, A., Bray, F., Center, M.M., et al., 2011. Global cancer statistics. CA Cancer J. Clin., 61(2):69-90.

http://dx.doi.org/10.3322/caac.20107

Ju, M.J., Qiu, S.J., Fan, J., et al., 2009a. Peritumoral activated hepatic stellate cells predict poor clinical outcome in hepatocellular carcinoma after curative resection. Am. J. Clin. Pathol., 131(4):498-510. http://dx.doi.org/10.1309/AJCP86PPBNGOHNNL

Ju, M.J., Qiu, S.J., Gao, Q., et al., 2009b. Combination of peritumoral mast cells and T-regulatory cells predicts prognosis of hepatocellular carcinoma. Cancer Sci., 100(7):1267-1274.

http://dx.doi.org/10.1111/j.1349-7006.2009.01182.x

Kobayashi, N., Hiraoka, N., Yamagami, W., et al., 2007. $\mathrm{FOXP}^{+}$regulatory $\mathrm{T}$ cells affect the development and progression of hepatocarcinogenesis. Clin. Cancer Res., 13(3):902-911.

http://dx.doi.org/10.1158/1078-0432.CCR-06-2363

Lan, X,P., Chen, Y.G., Wang, Z., et al., 2015. mmunotherapy of DC-CIK cells enhances the efficacy of chemotherapy for solid cancer: a meta-analysis of randomized controlled trials in Chinese patients. J. Zhejiang Univ.-Sci. B (Biomed. \& Biotechnol.), 16(2):743-756. http://dx.doi.org/10.1631/jzus.B1500003

Lin, S.Z., Chen, K.J., Xu, Z.Y., et al., 2013. Prediction of recurrence and survival in hepatocellular carcinoma based on two Cox models mainly determined by FoxP $3^{+}$ 
regulatory T cells. Cancer Prev. Res. (Phila), 6(6):594-602. http://dx.doi.org/10.1158/1940-6207.CAPR-12-0379

Mathai, A.M., Kapadia, M.J., Alexander, J., et al., 2012. Role of Foxp3-positive tumor-infiltrating lymphocytes in the histologic features and clinical outcomes of hepatocellular carcinoma. Am. J. Surg. Pathol., 36(7):980-986. http://dx.doi.org/10.1097/PAS.0b013e31824e9b7c

Ormandy, L.A., Hillemann, T., Wedemeyer, H., et al., 2005. Increased populations of regulatory $\mathrm{T}$ cells in peripheral blood of patients with hepatocellular carcinoma. Cancer Res., 65(6):2457-2464. http://dx.doi.org/10.1158/0008-5472.CAN-04-3232

Peng, G., Li, S., Wu, W., et al., 2008. Circulating CD4 $4^{+}$CD25 regulatory $\mathrm{T}$ cells correlate with chronic hepatitis $\mathrm{B}$ infection. Immunology, 123(1):57-65. http://dx.doi.org/10.1111/j.1365-2567.2007.02691.x

Poon, R.T.P., Fan, S.T., Wong, J., 2000. Risk factors, prevention, and management of postoperative recurrence after resection of hepatocellular carcinoma. Ann. Surg., 232(1): 10-24. http://dx.doi.org/10.1097/00000658-200007000-00003

Sakaguchi, S., 2000. Regulatory T cells: key controllers of immunologic self-tolerance. Cell, 101(5):455-458. http://dx.doi.org/10.1016/S0092-8674(00)80856-9

Sasaki, A., Tanaka, F., Mimori, K., et al., 2008. Prognostic value of tumor-infiltrating FoxP $3^{+}$regulatory $\mathrm{T}$ cells in patients with hepatocellular carcinoma. Eur. J. Surg. Oncol., 34(2):173-179. http://dx.doi.org/10.1016/j.ejso.2007.08.008

Schoenleber, S.J., Kurtz, D.M., Talwalkar, J.A., et al., 2009. Prognostic role of vascular endothelial growth factor in hepatocellular carcinoma: systematic review and metaanalysis. Br. J. Cancer, 100(9):1385-1392. http://dx.doi.org/10.1038/sj.bjc.6605017

Shen, S.L., Liang, L.J., Peng, B.G., et al., 2011. Foxp3 ${ }^{+}$ regulatory $\mathrm{T}$ cells and the formation of portal vein tumour thrombus in patients with hepatocellular carcinoma. Can. J. Surg., 54(2):89-94. http://dx.doi.org/10.1503/cjs.028009

Shevach, E.M., 2002. CD4 ${ }^{+} \mathrm{CD} 25^{+}$suppressor T cells: more questions than answers. Nat. Rev. Immunol., 2(6):389-400. http://dx.doi.org/10.1038/nri821

Stross, L., Gunther, J., Gasteiger, G., et al., 2012. Foxp3 ${ }^{+}$ regulatory $\mathrm{T}$ cells protect the liver from immune damage and compromise virus control during acute experimental hepatitis B virus infection in mice. Hepatology, 56(3): 873-883. http://dx.doi.org/10.1002/hep.25765

Tierney, J.F., Stewart, L.A., Ghersi, D., et al., 2007. Practical methods for incorporating summary time-to-event data into meta-analysis. Trials, 8(1): 16 . http://dx.doi.org/10.1186/1745-6215-8-16
Unitt, E., Rushbrook, S.M., Marshall, A., et al., 2005. Compromised lymphocytes infiltrate hepatocellular carcinoma: the role of T-regulatory cells. Hepatology, 41(4):722-730.

http://dx.doi.org/10.1002/hep.20644

Wang, F., Jing, X., Li, G., et al., 2012. Foxp3 ${ }^{+}$regulatory $\mathrm{T}$ cells are associated with the natural history of chronic hepatitis B and poor prognosis of hepatocellular carcinoma. Liver Int., 32(4):644-655. http://dx.doi.org/10.1111/j.1478-3231.2011.02675.x

Will, B., Steidl, U., 2014. Stem cell fate regulation by dynein motor protein Lis1. Nat. Genet., 46(3):217-218. http://dx.doi.org/10.1038/ng.2902

$\mathrm{Xu}, \mathrm{D} ., \mathrm{Fu}, \mathrm{J} ., \mathrm{Jin}, \mathrm{L}$, et al., 2006. Circulating and liver resident $\mathrm{CD} 4{ }^{+} \mathrm{CD} 25^{+}$regulatory $\mathrm{T}$ cells actively influence the antiviral immune response and disease progression in patients with hepatitis B. J. Immunol., 177(1):739-747. http://dx.doi.org/10.4049/jimmunol.177.1.739

Zhou, J., Ding, T., Pan, W., et al., 2009. Increased intratumoral regulatory $\mathrm{T}$ cells are related to intratumoral macrophages and poor prognosis in hepatocellular carcinoma patients. Int. J. Cancer, 125(7):1640-1648. http://dx.doi.org/10.1002/ijc.24556

Zhou, L., Fu, J.L., Lu, Y.Y., et al., 2010. Regulatory T cells are associated with post-cryoablation prognosis in patients with hepatitis B virus-related hepatocellular carcinoma. $J$. Gastroenterol., 45(9):968-978. http://dx.doi.org/10.1007/s00535-010-0243-3

\section{中文概要}

题 目: 调节性 $\mathbf{T}$ 细胞在肝癌患者中的预后意义

目 的: 通过系统性文献检索和 meta 分析评估调节性 $\mathrm{T}$ 细胞与肝癌患者生存时间的相关性。

创新点: 针对调节性 $\mathrm{T}$ 细胞在肝癌患者中的预后意义进行 系统性文献综述和分析, 进一步证实其可以作为 预测肝癌预后的指标。

方 法: 在 MEDLINE 和 Embase 数据库中搜索相关文献, 提取数据并对文献质量进行评估。有 18 项研究 纳入到最后的分析, 包括的肝癌患者多数为乙肝 病毒感染。利用 meta 分析的方法对相关结果进行 合并分析。

结 论: 本实验证实, 肿瘤组织内调节性 $\mathrm{T}$ 细胞与肝癌患 者预后显著相关, 调节性 $\mathrm{T}$ 细胞数量高的患者生 存时间和无瘤生存期均较短。同样, 外周循环调 节性 $\mathrm{T}$ 细胞数量与肝癌患者生存期也呈负相关。

关键词: 肝细胞肝癌; 调节性 $\mathrm{T}$ 细胞; 预后 